\title{
A crise do diagnóstico em psiquiatria e os manuais diagnósticos
}

\section{The crisis of diagnosis in psychiatry and the diagnostic manuals}

Discute-se o caminho percorrido pela psiquiatria através dos manuais diagnósticos até o momento atual, com o projeto do Research Domain Criteria (RDoC), que busca, eliminando a subjetividade, alcançar uma excelência terapêutica na psiquiatria. São apresentadas quatro perspectivas para se pensar o diagnóstico das doenças psiquiátricas: as doenças que são descritas nos manuais de psiquiatria e que encontram correspondentes em pacientes na prática clínica; os transtornos que foram descritos nos manuais de psiquiatria e que geraram diagnósticos imprecisos; as síndromes culturais e o sofrimento psíquico que é estruturado de forma subjetiva e individual. Conclui-se que uma abordagem terapêutica deve levar em conta a natureza complexa do sofrimento psíquico, gerando mais cautela e individualização no que diz respeito às abordagens terapêuticas.

Palavras-chave: Psiquiatria, diagnóstico, fenomenologia, subjetividade

* Escola Bahiana de Medicina e Saúde Pública (Salvador, BA, Br). 


\section{Introdução}

A semiologia médica é a disciplina que fundamenta o método clínico na medicina, sendo então o instrumento através do qual se afirma ou se afasta uma doença e com o qual se constrói um diagnóstico. O exercício do método clínico se baseia na habilidade do médico em se apropriar das queixas e das manifestações da doença no paciente, podendo complementar a avaliação por meio de exames clínicos, laboratoriais ou de imagem para auxiliar na definição do diagnóstico. Na semiologia médica estudam-se os sinais e os sintomas das doenças, sendo que os sinais correspondem ao que pode ser percebido por outra pessoa sem o relato ou comunicação do paciente, enquanto os sintomas se referem às queixas subjetivas relatadas pelo enfermo (Dalgalarrondo, 2009). A propedêutica clínica se encarrega de criar meios para identificar os sinais que são considerados manifestações objetivas das doenças, tais como inspeção, palpação, percussão, ausculta, percussão auscultada ou teste de reflexos. A propedêutica armada, por seu turno, mune-se de exames laboratoriais, anatomopatológicos e exames de imagem, buscando sempre otimizar a captura dos sinais considerados objetivos das doenças. A propedêutica específica da psiquiatria é a psicopatologia, composta pelo exame psíquico, relato "objetivo" feito pelos familiares ou acompanhantes e o relato subjetivo das queixas do paciente. São exemplos de sinais de doença mental certas alterações na fisionomia do paciente, os tiques, as estereotipias, os risos imotivados, o bloqueio de pensamento, a catatonia, os delírios, as mussitações, a agitação psicomotora etc. O sintoma, 
por seu turno, que é a queixa relatada pelo paciente e que só ele consegue perceber, se refere às sensações vividas e descritas, tais como dores, tonturas, palpitações, medo, alucinações, percepções, tristeza etc. Todo encontro entre médico e paciente, cumpre ressaltar, se dá no espaço intersubjetivo, de modo que as queixas relatadas, que norteiam a avaliação subsequente e a hipótese diagnóstica, podem variar a depender do encontro estabelecido.

$\mathrm{Na}$ construção da hipótese diagnóstica, cabe ao médico buscar a descrição dos sintomas através do relato do paciente/familiar e a evidência de sinais pela observação clínica, bem como decifrar o significado de ambos em relação ao diagnóstico. O médico deverá discernir na sua escuta das queixas subjetivas, das queixas dos familiares e do exame clínico/psíquico aquilo que é relacionado com a suposta doença do que não é importante para o diagnóstico. Deverá, portanto, filtrar das queixas relatadas aquilo que de fato é indicativo da doença, discriminando se o sintoma referido tem como significado uma doença, para que a expressão das queixas do paciente não confunda, em vez de elucidar o diagnóstico. Esse julgamento sobre quais são os sintomas importantes, assim como o direcionamento da coleta de dados, são elaboração do médico; o que faz com que a experiência clínica seja, desde a coleta de dados, suspeita diagnóstica e tratamento proposto, o encontro entre duas subjetividades. Esse encontro, que norteia a construção de uma suspeita diagnóstica, leva o médico a escolher dentre os sintomas relatados e os sinais percebidos aqueles que ele considera importantes para o diagnóstico. A expressão dos sintomas varia com a fase do desenvolvimento do indivíduo, com a cultura, com o acesso à linguagem partilhada e também com aspectos próprios de grupos e de cada indivíduo (Good, 1977). A desconsideração, por parte do médico, de determinadas queixas e a ênfase em outras faz parte do quebra-cabeças que será montado. O diagnóstico clínico é, portanto, antes de tudo, um processo intersubjetivo, no qual o médico tem a função de tradutor. Como dizem os italianos, o tradutor é sempre um traidor (traduttore traditore). Por isso, o ideal perseguido através da propedêutica médica é eximir o máximo possível a subjetividade do processo diagnóstico, buscando com isso uma melhor precisão. A exclusão do subjetivo se dá de forma muito clara nas clínicas oncológicas e infectologia, por exemplo, para as quais a melhor especificação do agente patogênico (citologia do tumor ou isolamento do vírus) determina um tratamento mais individualizado. A psiquiatria, tendo como modelo a medicina de precisão praticada pela oncologia, busca, no seu momento atual, através das ideias em desenvolvimento pelo RDoC (Research Domain Criteria), do NIMH (National Institute of Mental Health), eximir a 
subjetividade ao máximo da avaliação psiquiátrica (Insel, 2014). A matriz proposta pelo RDoC considera que a subjetividade é um viés a ser evitado e vai buscar avaliar o doente, não pela descrição do mal-estar feita pelo mesmo, mas pela avaliação do que chamam de "construtos de comportamentos complexos observáveis", que será feita pelo uso da propedêutica armada, associada a testes neuropsicológicos e escalas de avaliação (Cuthbert \& Insel, 2013). Discutiremos neste artigo o caminho percorrido pela psiquiatria através dos manuais diagnósticos norte-americanos, até o momento atual, buscando compreender o percurso dessa disciplina médica, que é forjada nas suas entranhas pelo espaço intersubjetivo e que pretende, a partir do projeto $\mathrm{RDoC}$, declarar a morte desse espaço, correndo o risco de atestar o seu próprio óbito.

\section{O nascimento do DSM}

Dois autores de língua alemã, Kraepelin e Freud, ambos nascidos em 1856, são representantes de métodos de investigação distintos, que são basilares no pensamento classificatório e etiológico em psiquiatria. Kraepelin teve como referência a crença na existência de um mundo real (realismo), que existe e funciona independente da percepção humana, enfatizando que o psiquiatra clínico e o pesquisador devem descrever objetivamente o que realmente existe, ou seja, o que a natureza apresenta, assumindo que as doenças mentais são entes em si (Hoff, 2015). Ele defendeu o ponto de vista de que há diversas doenças mentais discretas, que se classificam como síndromes, que por sua vez são categoricamente diferentes entre si. A pedra angular da sua nosologia foi a observação clínica de longo prazo, sendo cético no que diz respeito aos aspectos subjetivos da doença mental, pois não poderiam ser estudados experimentalmente (Palm \& Möller, 2011). Ele observava e fazia anotações ao longo dos anos dos pacientes internados, tendo identificado duas grandes síndromes: a demência precoce, que levaria o doente a um quadro progressivo de deterioração mental e a psicose maníaco-depressiva, que se caracterizava por períodos de profunda depressão, intercalados por períodos de normalidade e de euforia (Ibid.). Freud, valendo-se de uma outra perspectiva, sugeriu a abordagem dos sintomas por meio da escuta do paciente, do significado do que se fala (Freud, 1909/1976a). Ele trabalhou sobretudo com a escuta de pacientes em consultório, que eram atendidos várias vezes por semana, durante um período longo de tempo. Freud (1916-1917/1976b) entendia que os sintomas, assim como os sonhos e os chistes, 
eram manifestações do inconsciente e entendia o aparelho psíquico como um conceito teórico e não como um órgão anatomicamente localizado. Os dois autores tiveram concepções distintas sobre o sintoma. Para Kraepelin, os sintomas identificados na observação clínica do paciente representam evidências de uma doença orgânica, à qual ele pretende se aproximar por meio de descrições precisas sobre o que observa no doente. Freud, diferentemente, toma o sintoma como signo, ou seja, o sintoma é uma formação do inconsciente, assim como o sonho, os chistes e os atos falhos, levando então ao sujeito do inconsciente pelo método interpretativo.

Os conceitos de Freud influenciaram muito a psiquiatria do início do século XX. Bleuler, psiquiatra da Suiça Alemã, autor central na história da psiquiatria dinâmica, incorporou as ideias da psicanálise a fim de construir um modelo explicativo para a etiologia das doenças mentais. A classificação nosológica descrita no Tratado de Psiquiatria, de Bleuler (1924), entende a doença mental como uma reação do aparelho psíquico aos eventos decorridos na trajetória da vida. A noção de doença mental como reação psicodinâmica será importante para compreender as duas primeiras edições do DSM (Manual Diagnóstico e Estatístico dos Transtornos Mentais, publicado pela Associação Americana de Psiquiatria), assim como a polarização que se estabeleceu entre a psiquiatria biológica e a psiquiatria psicodinâmica, impulsionada pelas ideias do NIMH, que foram traduzidas nas edições subsequentes do DSM (terceira e quarta com as suas revisões), até a quinta edição, que já nasceu com a sua sentença de morte anunciada pelo próprio NIMH.

A primeira classificação oficial dos EUA que contempla as doenças mentais apareceu no censo de 1840 e tinha duas categorias: insanidade e idiotia. Em 1880 uma classificação oficial elenca sete categorias: mania, melancolia, monomania, paresia, demência, dipsomania e epilepsia. Em 1917, surgiu a primeira classificação voltada para a produção de estatísticas hospitalares. Após o final da Segunda Guerra Mundial, pela pressão trazida pelo contingente de veteranos de guerra, apareceu um sistema classificatório que atendia não apenas às exigências burocráticas, mas às exigências de grupos sociais. A classificação existente na época, a $6^{a}$ edição da classificação internacional das doenças (CID) — que era editada pela Organização Mundial da Saúde (OMS) com sede em Genebra, não contemplava as situações clínicas dos veteranos de guerra, como as síndromes cerebrais crônicas e as reações situacionais (Bezerra Jr., 2014). No contexto histórico do pós-guerra, duas referências foram importantes para definir o padrão da primeira edição do DSM nos EUA: a psiquiatria psicodinâmica influenciada pelas ideias da 
psicanálise, e o movimento social dos veteranos de guerra, pressionando para que o Estado financiasse a sua reinserção na sociedade. A primeira edição do DSM (o DSM-I), publicada em 1952, trazia um total de 106 categorias, que eram consideradas reações a situações existenciais para as quais o indivíduo não seria capaz de oferecer uma resposta adequada, sendo divididas em transtornos neuróticos, psicóticos e de caráter. Os sintomas apresentados pelos doentes eram considerados uma expressão de conflitos subjacentes, de modo que a falência na adaptação às exigências da vida podia se expressar em reações de maior ou menor gravidade, gerando sintomas neuróticos ou psicóticos (Bezerra Jr., 2014). Nas duas primeiras edições do DSM, considera-se que os transtornos psiquiátricos existem ao longo de um espectro que emana das mesmas raízes, ou seja, de conflitos intrapsíquicos. No DSM-I e no DSM II, os transtornos eram divididos entre os associados a perturbações orgânico cerebrais e os demais que ocorrem sem perturbação orgânica primária.

A suposta neutralidade científica do DSM inexiste desde a sua fundação, que foi engendrada não por uma descoberta científica, mas pelo movimento social dos veteranos de guerra, que exigiram uma classificação que possibilitasse a sua reinserção social, salvaguardando os direitos e a dignidade extirpados pela guerra. Na década de 1970 o movimento social dos homossexuais também interferiu na suposta neutralidade e cientificidade do manual, já que esse grupo, enquanto organização social, exigiu que a sua condição fosse reconhecida fora do que consideravam "a codificação repressora da psiquiatria" (McCommon, 2006). Representantes e simpatizantes do movimento gay invadiram o congresso da Associação Psiquiátrica Americana (APA) impedindo a entrada de psiquiatras e levantando uma discussão que resultou, após trabalhos de uma comissão e votação de membros da APA, na retirada da categoria diagnóstica "Homossexualismo" da sétima impressão do DSM-II (McCommon, 2006).

\section{A polarização na psiquiatria, ou o surgimento do DSM-III}

Na segunda metade da década de 1970 a validade das categorias diagnósticas em psiquiatria vinha sendo criticada. As classificações da CID e do DSM foram duramente criticadas pela sua baixa validade e porque não eram adequadas para classificar os pacientes que procuravam tratamento. Strauss et al. (1979) partiram da hipótese que existia uma grande incongruência entre o sistema classificatório disponível e a forma como os pacientes se apresentam 
na prática clínica. Os autores descreveram 25 sintomas hipotéticos de 100 pacientes arquetípicos que deveriam ter o diagnóstico de depressão neurótica, depressão psicótica, esquizofrenia paranoide e mania. Depois, pacientes em primeira admissão em serviços de psiquiatria foram avaliados através dos mesmos 25 sintomas. Houve uma diferença importante na distribuição das características dos pacientes reais em relação aos arquetípicos. Alguns pacientes reais se localizavam no mapa em posições semelhantes aos dos pacientes arquetípicos, mas a maioria dos pacientes reais não se encaixava nas categorias arquetípicas, pois tinha baixos escores de diferentes sintomas, em vez dos altos escores de poucos sintomas dos pacientes arquetípicos. Uma série de outras críticas ao modelo conceitual do diagnóstico difundido pelas duas primeiras edições do DSM, notadamente a sua vinculação à concepção psicodinâmica da psicanálise, engendraram a formatação da terceira edição do manual.

O contexto que delineia o surgimento do DSM-III são críticas aos manuais diagnósticos que o precederam, a incipiente psicofarmacologia e o surgimento dos critérios Feighner, desenvolvidos na Universidade de Washington e, em seguida, pelo RDC (Research Diagnostic Criteria), desenvolvido pelo NIMH. A terceira edição do Diagnostic and Statistical Manual of Mental Disorders (DSM-III) (APA, 1980) só incluía etiologia quando se relacionava com doenças de origem claramente orgânica, evitando o conceito de reação psicodinâmica; o termo reação, aliás, já vinha sendo evitado no DSM-II. Cada diagnóstico aparecia com uma série de critérios de inclusão e exclusão, bem como critérios de duração, em vez das descrições mais gerais das versões anteriores. As mudanças ocorridas a partir do DSM-III foram propaladas como uma estratégia para permitir a comunicação entre os profissionais de orientações teóricas diversas. As mudanças no manual traziam em seu bojo um retorno ao projeto de Kraepelin, da crença na organicidade da doença mental (em oposição à crença na psicodinâmica das edições I e II), apoiados e guiados pela crescente importância dada às descrições biológicas que foram muito valorizadas pelas intervenções psicofarmacológicas iniciadas na década de 1950. Definindo-se como agnóstico e recusando de forma explícita o fundamento etiológico para o diagnóstico, o DSM-III assumia que as doenças psiquiátricas seriam entidades biológicas discretas, deixando subentendido a crença que cada doença teria causas e mecanismos fisiopatológicos distintos de base biológica (Bezerra Jr., 2014). Apesar desse ideário implícito, o DSM-III definiu as doenças a partir de uma lista arbitrária de critérios de inclusão e exclusão que fixam o número, a duração e a qualidade dos traços a serem observados na construção do diagnóstico, sem uma base clínica evidente para essa classificação, muito menos uma 
observação clínica evolutiva. Os diagnósticos passaram a ser agregados uns aos outros, com a noção de comorbidade servindo à pesquisa, estudos epidemiológicos e ensaios clínicos.

A literatura jornalística, embasada por depoimentos de psiquiatras envolvidos na elaboração do manual, contesta a cientificidade da terceira edição do DSM, sugerindo que as suas categorias foram forjadas para suprir prioritariamente as necessidades da pesquisa clínica. Stossel (2014) descreve elementos que, se não foram determinantes, podem ter contribuído para a elaboração de duas categorias diagnósticas emergentes no DSM-III: a Síndrome do Pânico e o Transtorno de Ansiedade Generalizada. A Síndrome do Pânico, descrita por Donald Klein, baseou-se na eficácia trazida pelo uso da imipramina a pacientes que apresentavam crises episódicas de ansiedade. A imipramina, uma droga nova na época, foi usada por Klein com a maioria dos 200 doentes internados no Hospital Hillside em Nova York, sendo que levou à cessação dos sintomas de 14 pacientes que tinham crises agudas episódicas de ansiedade (Stossel, 2014). Da melhora clínica trazida pela imipramina, especificamente de um paciente que "entrava em pânico" durante as crises, surgiu dedutivamente a categoria diagnóstica "Síndrome do Pânico". O raciocínio de Klein partia da negação da premissa de que a ansiedade seria uma reação psicológica a estressores, variando de uma ansiedade branda que levaria a comportamentos neuróticos, enquanto a grave geraria esquizofrenia ou a psicose maníaco-depressiva (Stossel, 2014). Klein, na observação do caso do seu paciente "em pânico", deduziu que a ideia predominante na época de que a psicopatologia derivava da ansiedade (que por sua vez era causada por conflito intrapsíquico) era falsa; pois os seus achados com a imipramina (uma droga) o levaram a supor que o que causava o pânico era uma disfunção biológica. Enquanto a categoria "síndrome do pânico" pode ter tido a sua origem no raciocínio de um psiquiatra brilhante, a categoria "transtorno de ansiedade generalizada" parece ter tido origem em um jantar. Sheehan, eminente psiquiatra que fazia parte da força tarefa para elaboração do manual, assinala que durante um jantar na década de 1970, no qual estavam presentes vários outros psiquiatras componentes da força tarefa do DSM-III, conversaram sobre um colega que não sofria de ataques de pânico, mas que estava constantemente preocupado. Entre pilhérias e garrafas de vinho, questionaram-se como haveriam de classificá-lo, surgindo então o termo "ansiedade generalizada". Então, com um brinde, batizaram essa doença, sobre a qual coletam-se dados até o presente (Stossel, 2014). Esses dois exemplos indicam que, de forma muito diferente de como foram elaboradas 
as categorias diagnósticas de Kraepelin durante anos de acompanhamento de pacientes hospitalizados, sobretudo pela observação do curso da doença, algumas categorias diagnósticas do DSM-III foram cunhadas, por um lado, de forma mais displicente, no caso do transtorno de ansiedade generalizada e, por outro, dedutivamente, pelo efeito de um psicofármaco (a imipramina) no caso da síndrome do pânico.

\section{Os efeitos do DSM-III e DSM-IV na clínica}

As críticas à psiquiatria psicodinâmica que serviram de base às duas primeiras versões do DSM, que supunha que o sofrimento psíquico seria uma reação psicológica ao estresse, foi dando lugar à crença na origem biológica dos chamados transtornos mentais. A partir da sua terceira edição as categorias do manual diagnóstico norte-americano foram sendo reificadas, a ponto das doenças psiquiátricas se resumirem ao que está descrito no DSM, ou seja, indicadores da doença foram tomados como a doença em si (Kendler, 2016). Um bom critério diagnóstico para o DSM-III deveria ser sucinto e requerer o mínimo de inferência, o que empobrece a psicopatologia, afetando a clínica e a pesquisa, pois não corresponde à realidade de fenômenos que são de difícil acesso e experienciados pelo sujeito de várias formas (Ibid.). Apesar desse empobrecimento psicopatológico, o DSM-III e suas versões subsequentes até o DSM-5, tornaram-se, ao longo dos últimos 36 anos, manuais hegemônicos para a classificação da doença mental, influenciando inclusive na classificação da OMS, a CID (Classificação Internacional das Doenças). Algumas hipóteses são levantadas buscando compreender a razão dessa hegemonia. Nos EUA o sistema de saúde privatizado exige o diagnóstico para acesso aos cuidados. Com a substituição da regulação do Estado pela regulação do mercado no campo da saúde, o espaço ficou aberto para a entrada do complexo médico industrial (CMI), que por sua vez buscou se legitimar através da academia médica (Ramos, 2014). O CMI se refere a artigos do setor produtivo industrial, de base química e biológica (medicações, hemoderivados etc.), mecânica (próteses, camas hospitalares etc.), eletrônica (equipamentos diagnósticos), setor de prestação de serviço (hospitais e ambulatórios), além dos prestadores de serviço (médicos, enfermeiros, fisioterapeutas, psicólogos, gestores, pesquisadores etc.) (Oliveira Mendonça \& Camargo, 2012). Na cardiologia, por exemplo, o conceito de hipertensão ampliou muito o uso de anti-hipertensivos e a ideia de hiperlipidemia como fator de risco para doença 
cardiovascular abriu um amplo mercado para as estatinas. A nova estratégia diagnóstica do DSM-III, ampliando as possibilidades de diagnóstico e retirando a causalidade do campo da psicodinâmica, abriu espaço para o tratamento farmacológico das doenças que eram do campo da psicoterapia. $\mathrm{O}$ DSM-III foi adotado pelo CMI norte-americano para a realização dos ensaios clínicos randomizados e passou a ser uma referência obrigatória para qualquer discussão válida no campo da saúde mental, em qualquer país do Ocidente (Ramos, 2014).

A retirada progressiva da etiologia psicodinâmica no campo de ação da psiquiatria ampliou na prática as possibilidades de intervenção farmacológica. Se essa não foi a intenção primeira do manual, como reação secundária o modelo se encaixou perfeitamente nas regras de ampliação do mercado do complexo médico industrial (Ibid.). O modelo biomédico passou a ser o discurso hegemônico que formou toda uma geração de psiquiatras, que por sua vez reproduziram o mesmo modelo junto às gerações seguintes (Braken et al., 2012). Psiquiatras e neurologistas foram convidados pela indústria farmacêutica, nos últimos 10 anos, a proferir conferências sobre o diagnóstico do transtorno de déficit de atenção e hiperatividade (TDAH) em escolas, de modo a difundir o conceito para além dos limites dos consultórios médicos. Além disso, revistas leigas e jornais circulam notícias sobre os avanços das neurociências e a eficácia das drogas no tratamento daquele transtorno, expandindo o mercado e gerando mais indivíduos diagnosticados, agora com as suspeitas feitas por professores, pelos pais e pelos próprios pacientes.

A expansão do diagnóstico psiquiátrico tem gerado demandas em consultório características das últimas décadas, desde adolescentes pré-vestibulandos que precisam de um desempenho para além do que produzem para concorrer às vagas nos cursos de medicina, até pessoas que concorrem a empregos através de concursos públicos e que precisam de um desempenho acima das suas possibilidades habituais. A clínica contemporânea é composta ainda por crianças e adolescentes que não conseguem um desempenho escolar e social associado à falta de interesse pelos estudos, a pais que estão ausentes porque precisam trabalhar, ou a pais que negligenciam os filhos em razão do seu próprio sofrimento. Essas crianças não se concentram nos estudos, mas se focam obsessivamente em jogos eletrônicos, filmes ou séries de TV. Como "efeito colateral" dessa nova prática aparecem as pessoas com distúrbio do sono, como inversão do ciclo sono-vigília, ou com insônia inicial e sonolência diurna. Essas são descrições da minha prática clínica do que Bezerra Jr. (2014) chamou de "sintomas do seu tempo", pois algumas categorias 
psiquiátricas revelam, de forma deslocada, a verdade de conflitos de uma sociedade que não se deixam apreender imediatamente pela consciência.

\section{O RDoC e a crença na supremacia biológica}

O NIMH (National Institute of Mental Health) vem questionando de forma incisiva a validade diagnóstica do DSM-5, uma vez que os diagnósticos ali contidos não representam síndromes compatíveis com pacientes reais, crítica aliás que vem acompanhando esse manual diagnóstico desde a sua segunda edição, pelo próprio NIMH, que foi o principal artífice do DSM-III. O Research Domain Criteria (RDoC) parte da ideia de que há mais fronteiras, sobretudo neurobiológicas, entre as doenças, do que aquelas baseadas na psicopatologia, ou seja, na descrição de sintomas clínicos (Cuthbert \& Insel, 2013). A ideia central da matriz do RDoC é guiada pela crença de que existem mecanismos neurobiológicos e biocomportamentais subjacentes às doenças mentais e que o desvelamento desses mecanismos levará a tratamentos biológicos definitivos (Insel, 2014). Insel chama atenção para que o diagnóstico pelos manuais se limita aos sintomas, e o tratamento se resume ao alívio dos sintomas, não alcançando a cura ou intervenção preventiva, sendo que o objetivo do RDoC é integrar vários níveis de dados para desenvolver uma abordagem nosológica baseada na fisiopatologia. O RDoC, portanto, é a versão do século XXI do RDC, que impulsionou o DSM-II em direção ao DSM-III (Ibid.). Cumpre lembrar que esse redirecionamento rumo ao DSM-III, que se "aprimorou" no DSM-III-R, DSM-IV e DSMIV-TR desembocou na atual DSM-5, que o próprio NIMH agora rejeita como destituído de validade científica.

$\mathrm{O}$ RDoC pretende integrar diferentes níveis de informação (genoma, avaliação neuropsicológica e dados dos pacientes coletados através de escalas de autorrespostas), eliminando a subjetividade e a introspecção na avaliação, de modo que os estados mentais de qualquer natureza e complexidade sejam avaliados a partir do comportamento observável (NIMH, 2017). A estrutura do RDoC é constituída por uma matriz na qual as linhas representam construtos referentes a uma dimensão funcional específica do comportamento (Cuthbert \& Insel, 2014). Esses construtos são agrupados no que chamam de "domínios de hierarquia elevados do funcionamento", que refletem o conhecimento atual sobre os sistemas da emoção, cognição, motivação e comportamento social (NIMH, 2017). Os construtos são caracterizados pelos genes, moléculas e circuitos cerebrais usados para mensurá-los e são classificados em 
cinco: sistemas de valência negativa, sistemas de valência positiva, sistema cognitivo, sistema de processos sociais e sistema de regulação (Ibid.). Os sistemas de valência negativa são responsáveis por respostas a situações ou contextos aversivos, sendo representados por medo, ansiedade e perda (Ibid.). Os sistemas de valência positiva são responsáveis por respostas a contextos ou situações motivacionais positivas, tais como busca de recompensa, comportamento consumatório e aprendizado da recompensa. O sistema cognitivo envolve atenção, percepção, memória declarativa, linguagem, controle cognitivo e memória de trabalho. O sistema de processos sociais é o sistema que faz a mediação da resposta a configurações interpessoais, incluindo percepção e interpretação das ações do outro. O sistema excitação/regulação é um continuum que vai da vigília a estados de baixa excitação como sono, anestesia e coma. As unidades de análise de cada sistema são os circuitos (medidos por neuroimagem, validado por modelo animal ou neuroimagem funcional), fisiologia (medidas que são índices de certos construtos, mas que não necessariamente tocam os circuitos de forma direta, como batimentos cardíacos, nível de cortisol), comportamento (refere-se a tarefas comportamentais, como tarefas de memória de trabalho; ou observação de comportamento, como observação de bebê) e autorresposta, que são as respostas às escalas de avaliação (Ibid.).

A proposta do RDoC se espelha na oncologia, buscando bases biológicas seguras para as doenças mentais e busca transformar o diagnóstico pela incorporação dos conhecimentos da genética, neuroimagem, ciência cognitiva e outros níveis de informação com o fim de estabelecer as bases para um novo sistema classificatório (Cuthbert \& Insel, 2014). Apesar do RDoC não ter criado nenhuma proposta classificatória, a ideia é buscar classificar os transtornos mentais com base em dimensões observáveis do comportamento e em medidas neurobiológicas, priorizando a exclusão da experiência subjetiva, que obstaculizaria a explicação do processo subjacente. $\mathrm{Na}$ concepção da RDoC o comportamento seria apreendido através da psicometria, com o instrumental da neuropsicologia, sendo os seus correlatos biológicos apreendidos pela propedêutica armada, medicina molecular e estudo do genoma. Não há espaço para a subjetividade na matriz do RDoC, uma vez que a fenomenologia e as narrativas da experiência subjetiva vividas não são contempladas. Difícil é imaginar de que forma o cérebro humano poderia ser acessado e explicado sem que a expressão dos processos biológicos seja determinada, ou pelo menos perpassada pela expressão subjetiva, para não dizer humana, das experiências anímicas, uma vez que a percepção e vivência das experiências, 
associadas ao genótipo é que conforma o fenótipo. Avançar no conhecimento científico ocultando fatos ou maquiando o objeto de pesquisa pode resultar, mais uma vez, no encontro com modelos arquetípicos que não correspondem ao que é visto na prática clínica.

\section{O lugar da subjetividade}

A perspectiva fenomenológica, da psiquiatria baseada na narrativa, advoga a adoção de métodos de investigação capazes de fazer avançar o conhecimento dos determinantes biológicos e, ao mesmo tempo, elucidar as dimensões da experiência subjetiva que não são redutíveis à biologia. A psiquiatria está profunda e irreversivelmente ligada ao mundo mental, e essa ligação não é uma contingência, pois a psiquiatria está intrinsecamente ligada ao mental e à experiência subjetiva (Kendler, 2005). O trabalho clínico implica a avaliação e interpretação constantes da experiência subjetiva, já que o seu objetivo e objeto são o sofrimento humano. Cada fenômeno ou manifestação, diz Jaspers, tem nuanças variadas conforme os diversos doentes, não consistindo apenas no desenvolvimento mais ou menos pleno e sim no fato que, dentro do mesmo desenvolvimento, todos os processos psíquicos têm modificações que decorrem em parte de individualidade diversa, em parte de várias alterações psíquicas, que podem ser as mais gerais (Jaspers, 1913/1985). Jaspers defende, ao contrário de Kraepelin, que não existem unidades nosológicas em psicopatologia. Para ele os quadros típicos de psicose correspondem a um pequeno número de casos, sendo que a grande maioria reflete a multiplicidade e singularidade das doenças mentais. Kendler (2016) nega que as doenças psiquiátricas tenham essências, mas nascem de uma rede de causas, sintomas e signos. Defende que se enfatize os "tipos psiquiátricos", em oposição aos "tokens" de doenças psiquiátricas descritas nos manuais diagnósticos.

A psicopatologia diz respeito à descrição dos sintomas que levam o psiquiatra ao diagnóstico, mas, também, busca descrever a experiência do paciente e a relação dessa experiência com o paciente e com o mundo (Stanghellini \& Broome, 2014). Não se trata, portanto, apenas da descrição de sintomas para a composição de diagnósticos através de checklists, mas a descrição de todo o campo de experiência da pessoa. O foco nos sintomas que levam a um diagnóstico específico termina por desviar a atenção da experiência vivida pelo paciente, desviando o foco da própria psiquiatria que é a subjetividade do fenômeno vivido pelo paciente. Numa vertente diversa daquela 
proposta pelo RDoC, Stanghellini e Broome (2014) defendem que as expressões e os comportamentos são importantes, mas menos específicos do que a experiência vivida, por isso a ênfase na subjetividade é a única forma de respeitar o fenômeno vivido pelo paciente.

Seguindo a perspectiva de avaliar os processos mentais segundo a sua apresentação concreta, Northoff e Stanghellini (2016) propõem a pesquisa em neurociência a partir de exames de imagem do cérebro, sem estímulo externo e baseada no relato da experiência vivida pelo paciente. O cérebro e a mente são conformados pela experiência, que por sua vez ocorrem no contexto cultural no qual a pessoa se desenvolve e vive (Han \& Northoff, 2008). Estudos de neuroimagem em perspectiva transcultural sugerem que o contexto cultural influencia a atividade neural que está na base das funções cognitivas (Han \& Northoff, 2008). Influências socioculturais têm um papel importante no desenvolvimento e nos processos cerebrais ao longo da vida, assim como os processos biológicos que influenciam as respostas cerebrais (Kirmayer \& Crafa, 2014). O ambiente que interfere no desenvolvimento do homem e nas suas vicissitudes não se resume a agentes físicos mensuráveis, mas, também, a aspectos perceptivos cuja ponderação quantitativa pode não ser tão evidente. Por exemplo, crianças mantidas em isolamento social prolongado exibem desenvolvimento cerebral anormal e não adquirem habilidades motoras básicas e linguagem (Watts-English et al., 2006; Ress, 2008). O cérebro se adapta ao ambiente sociocultural e o seu funcionamento pode ser melhor compreendido em relação às oportunidades e demandas do contexto (Kirmayer \& Crafa, 2014). A identificação de sistemas dinâmicos fornece ao clínico a possibilidade de ampliar a abordagem dos problemas em termos de uma causalidade circular, que inclua não apenas os circuitos neurais, mas, também, aspectos pessoais e sociais (Kirmayer \& Sartorius, 2009). É tão importante perguntar o que muda do cérebro em resposta a relações de confiança com alguém, quanto como uma alteração específica da amígdala cerebral influencia na interação social (Phan et al., 2006; Lupien et al., 2011).

\section{Comentários finais}

Existem particularidades das doenças psiquiátricas que as distinguem ontologicamente das outras doenças crônicas: o funcionamento relacional do homem se dá no campo da subjetividade e é nesse campo que o sofrimento 
psíquico se constitui, havendo uma complexa e constante interação entre predisposição genética (conformação cerebral, raça, gênero etc.) e fatores ambientais, que incluem exposições ambientais a agentes físico-químicos (pesticidas, poluentes ambientais etc.), estressores que vão de violência física (agressão sexual ou física) e psicológica (pais verbalmente agressivos, rejeição das figuras de apego, rejeição e violência em escola etc.), até violência/estresse gerado por fatores culturais (como etnia negra ou árabe em países ocidentais, homossexualidade, gênero etc.). Há que se pensar ainda que fatores de proteção ambientais (alimentação, atividade física, relações afetivas, segurança econômica etc.) também interferem na relação entre a mente/cérebro e o ambiente. Todas essas interações são mediadas, ou atravessadas ou entrelaçadas pela subjetividade, que por sua vez é, também, composta e se compõe permanentemente pelos fatores ambientais e genéticos.

A definição sobre a doença psiquiátrica deve levar em conta o questionamento sobre o que é esse objeto e, também, o que é feito dele na prática clínica, o que necessariamente clama por uma discussão sobre que valores estão envolvidos na definição da doença e na prática de tratamento/cura. No que diz respeito à ontologia, trata-se de um objeto que deve ser perseguido na forma concreta como ele se apresenta no mundo real. A definição da doença mental depende de maneira fundamental do contexto sociocultural e histórico. A forma que o cérebro e as funções adaptativas são usadas dependem, também, do contexto sociocultural, do momento histórico e das trajetórias de desenvolvimento de cada sujeito. O funcionamento mental na saúde e na doença envolve adaptar-se e responder às demandas sociais, o que tem implicações para qualquer tipo de abordagem das neurociências em psiquiatria que almeje relevância clínica (Kirmayer \& Crafa, 2014).

Nesse sentido, podemos pensar a doença psiquiátrica em três perspectivas fundamentais. Primeiro, as doenças que são descritas nos manuais de psiquiatria e que encontram na prática clínica correspondentes em pacientes reais, a exemplo do autismo típico de Kanner e dos quadros clássicos de esquizofrenia. Essas doenças, assim como as síndromes genéticas (Síndrome de Down, Síndrome de Rubinstein-Taybi etc.), caracterizam-se por um suposto substrato biológico tão proeminente que o estar no mundo desses indivíduos é marcado de forma sui generis por esse substrato em todo o seu funcionamento adaptativo e subjetivo. No caso das síndromes genéticas o substrato biológico é tido como claramente definido, enquanto na esquizofrenia e no autismo esse substrato é incerto, mas presumido. Essas doenças têm estabilidade no tempo e no espaço, bem como apresentação clínica semelhante nos diferentes 
contextos socioculturais. Mesmo assim, os indivíduos que portam essas doenças apresentam-se de forma individualizada, com matizes de adaptação social muito variáveis a depender do contexto sociocultural e histórico. Segundo, alguns transtornos que foram descritos nos manuais de psiquiatria nas últimas décadas e que geraram diagnósticos imprecisos, pois constituem retratos instantâneos e parciais de sintomas e sinais, por um lado, inespecíficos e, por outro, sem limites claramente definidos com outros transtornos, a exemplo do chamado Transtorno de Déficit de Atenção e Hiperatividade, que define um complexo sintomático presente no autismo, nos transtornos de humor (depressão e bipolaridade), nos transtornos de ansiedade, no transtorno obsessivo compulsivo, podendo o déficit de atenção ainda ser um traço desadaptativo (no sentido de adaptação sociocultural frente às demandas sociais) como o são igualmente a dislexia e a discalculia. Terceiro, as chamadas síndromes culturais (culture-bound syndromes) que definem funcionamentos socioculturais desadaptativos, conformados pela interação entre o sofrimento subjetivo e características da cultura de origem, como é o caso da anorexia nervosa, alcoolismo e drogadição, jogo patológico, susto etc. Essas doenças podem ser consideradas patoplastias de um sofrimento que se conforma no 160 indivíduo a partir de elementos da cultura. Quarto, o sofrimento psíquico que é estruturado de forma subjetiva e individual, a partir da complexa interação entre um corpo biológico (com herança de ambos os genitores, e que não é estático, porque se modifica constantemente com a interação com o ambiente), as complexas interações com o ambiente físico que geram estresse oxidativo ou quelação (alimentos, poluentes ambientais), as complexas interações com a cultura, com o ambiente socioeconômico (gerando fatores ambientais protetores ou agressores) e com as relações interpessoais que atravessam a vida dos sujeitos (pais, irmãos, amigos e colegas, cônjuges etc.) e, portanto, a sua subjetividade, resultando em sujeitos mais ou menos adaptados, com sofrimentos engendrados por essa complexa interação de fatores.

É essa clínica polimorfa e complexa, que para fins práticos e comerciais é essencializada e reificada em diagnósticos parciais, que em geral não correspondem à demanda clínica do paciente, que compõe a prática diária do psiquiatra clínico. Ghaemi (2009) alerta para o risco de se deslocar a subjetividade para o limbo de um viés a ser evitado, gerando uma psiquiatria sem mente, que seria pouco relevante na medida em que desconsidera a experiência individual e a demanda daquele que busca tratamento. A prática clínica em psiquiatria implica um encontro entre duas subjetividades, devendo se resguardar a ideia de que, se a demanda do paciente não define por si só o que 
deve ser tratado, deve ser um norte para alcançar o sofrimento que aflige o paciente e/ou a sua família. Esta assertiva se debate com uma prática clínica voltada para diagnósticos parciais erigidos nas últimas versões do DSM e com a proposta futura de exclusão radical da subjetividade do RDoC. O paciente busca aplanar o seu sofrimento, enquanto o clínico busca uma categorização diagnóstica na qual ele possa essencializar aquele sujeito e dirigir o seu tratamento, em geral farmacológico. Aqui entra em campo que valores estão orientando a direção do tratamento que, apoiado no diagnóstico do DSM (ou no futuro diagnóstico do RDoC), centra-se na busca de uma medicação que possa tratá-lo. A despeito das supostas doenças descritas no DSM não disporem de tratamento médico definido, o clínico busca desesperadamente, na sua prática cotidiana, aproximações terapêuticas que possam gerar tratamentos médicos. Nesse sentido, o psiquiatra clínico vai constituindo uma prática terapêutica individualizada, tomando elementos terapêuticos disponíveis no seu arsenal farmacológico e físico (estimulação magnética e eletroconvulsoterapia) para tratar os casos particulares, sem que haja "evidência científica" que sirva de base para sua prática. Voltamos ao problema ontológico, que diz respeito ao que queremos e podemos tratar.

Ghaemi (2009) retoma a concepção de tipos ideais de Jaspers para que a natureza abstrata dos nossos diagnósticos seja relembrada, diante do fato concreto que os diagnósticos psiquiátricos em sua totalidade são conceituais, não correspondem a nenhuma entidade natural. Assim procedendo, podemos separar melhor os transtornos mentais com predominância de componentes genéticos ou ambientais, tentando identificar individualmente os fatores mais associados ao sofrimento psíquico daquele indivíduo, buscando assim o ideal do tratamento ultraindividualizado. Não se pode perder de vista que o reducionismo biológico ou comportamental não levará ao tratamento individualizado, porque o indivíduo humano é marcado e constituído pela subjetividade, que não pode, portanto, ser excluída do processo diagnóstico e nem terapêutico. Uma abordagem terapêutica honesta deve levar em conta a natureza complexa do sofrimento psíquico e das síndromes psiquiátricas, gerando, pois, mais cautela e individualização no que diz respeito às abordagens terapêuticas.

\section{Referências}

American Psychiatric Association. (1980). Diagnostic and Statistical Manual of Mental Disorders ( $3^{\text {rd }}$ ed.). Washington DC: American Psychiatric Press. 
Bezerra Jr., B. (2014). A psiquiatria contemporânea e seus desafios. In R. Zorzanelli, B. Bezerra Jr., \& J. F. Costa (Org.), A criação de diagnósticos da psiquiatria contemporânea. Rio de Janeiro, RJ: Garamond.

Bleuler, E. (1924). Tratado de Psiquiatria. Madrid: Calpe.

Bracken, P., Thomas, P., Timimi, S., Asen, E., Behr, G., Beuster, C., et al. (2012). Psychiatry beyond the current paradigm. British Journal of Psychiatry, 201, 430$-434$.

Cuthbert, B. N., \& Insel, T. R. (2013). Toward the future of psychiatric diagnosis: the seven pillars of RDoC. BMC Medicine, 11, 126.

Dalgalarrondo, P. (2009). Psicopatologia e semiologia dos transtornos mentais. Porto Alegre, RS: Artmed.

Freud, S. (1976a). Duas histórias clínicas: o pequeno Hans e o homem dos ratos. Rio de Janeiro, RJ: Imago. (Trabalho original publicado em 1909).

Freud, S. (1976b). Conferências introdutórias sobre psicanálise. Rio de Janeiro, RJ: Imago. (Trabalho original publicado em 1916-1917[1915-1917]).

Good, B. J. (1977). The heart of what's the matter the semantics of illness in Iran. Culture, medicine and psychiatry, 1(1), 25-58.

Ghaemi, S. N. (2009). Nosologomania: DSM \& Karl Jaspers' critique of Kraepelin. Philosophy Ethics Humanities Medicine, 4, 10.

Han, S., \& Northoff, G. (2008). Culture-sensitive neural substrates of human cognition: a transcultural neuroimaging approach. Nature Reviews Neuroscience, 9, 646-54.

Hoff, P. (2015). The Kraepelinian tradition. Dialogues in clinical neuroscience. Dialogues Clininal Neuroscience, 17, 31-41.

Insel, T.R. (2014). The NIMH Research Domain Criteria (RDoC) Project: precision medicine for psychiatry. American Journal of Psychiatry, 171, 395-7.

Jaspers, K. (1985). Psicopatologia Geral (2 ${ }^{\mathrm{a}}$ Ed., Samuel P. Reis, trad.). S. Paulo, SP/Rio de Janeiro, RJ/Belo Horizonte, BH: Atheneu. (Trabalho original publicado em 1913).

Kendler, K. S. (2005). Toward a philosophical structure for psychiatry. American Journal of Psychiatry, 162, 433-40.

Kendler, K. S. (2016). The phenomenology of major depression and the representativeness and nature of DSM criteria. American Journal of Psychiatry, $173,771-80$.

Kirmayer, L. J., \& Crafa, D. (2014). What kind of science for psychiatry? Frontiers in Human Neuroscience, 8, 435.

Kirmayer, L. J., \& Sartorius, N. (2009). Cultural models and somatic syndromes. In 
J. E. Dimsdale, Y. Xin, A. Kleinman et al. (Eds), Somatic presentations of mental disorders: refining the research agenda for DSM-V. Arlington, VA: American Psychiatric Association.

Lupien, S. J., Parent, S., Evans, A. C., Tremblay, R. E., Zelazo, P. D., Corbo, V. et al. (2011). Larger amygdala but no change in hippocampal volume in 10-year-old children exposed to maternal depressive symptomatology since birth. Proceedings of the National Acaddemy of Sciences U S A, 108, 14324-9.

McCommon, B. (2006). Antipsychiatry and the gay rights movement. Psychiatric Services, 57, 1809-1809.

National Institute of Mental Health (2017). Research Domain Criteria Matrix. Recuperado em 27 nov. 2017 de <https://www.nimh.nih.gov/research-priorities/ rdoc/constructs/rdoc-matrix.shtml $>$.

Northoff, G., \& Stanghellini, G. (2016). How to link brain and experience? Spatiotemporal psychopathology of the lived body. Frontiers in Human Neurosciece, 10, 76.

Oliveira Mendonça, A. L., \& Camargo Jr, R. (2012). Complexo médico-industrial/ financeiro: os lados epistemológico e axiológico da balança. Physis-Revista de Saúde Coletiva, 22(1), 215-238.

Palm, U., \& Möller, H. J. (2011). Reception of Kraepelin's ideas 1900-1960. Psychiatry and clinical neurosciences, 65(4), 318-325.

Phan, K. L., Fitzgerald, D. A., Nathan, P. J., \& Tancer, M. E. (2006). Association between amygdala hyperactivity to harsh faces and severity of social anxiety in generalized social phobia. Biological Psychiatry, 59, 424-9.

Ramos, F. (2014). In R. Zorzanelli, B. Bezerra Jr. \& J. F. Costa (Orgs.), A criação de diagnósticos da psiquiatria contemporânea. Rio de Janeiro, RJ: Garamond.

Rees, C. (2008). The influence of emotional neglect on development. Paediatric Child Health, 18, 527-34.

Stanghellini, G., \& Broome, M. R. (2014). Psychopathology as the basic science of psychiatry. British Journal of Psychiatry, 205, 169-70.

Stossel, S. (2014). Meus tempos de ansiedade: medo, esperança, terror e a busca da paz de espírito. São Paulo, SP: Companhia das Letras.

Strauss, J. S., Gabriel, K. R., Kokes R. F., Ritzler B. A., Van Ord, A., \& Tarana, E. (1979). Do psychiatric patients fit their diagnoses? Patterns of symptomatology as described with the biplot. Journal of Nervous Mental Disease. 167, 105-13.

Watts-English, T., Fortson, B. L., Gibler, N., Hooper, S. R., \& De Bellis, M.D. (2006). The psychobiology of maltreatment in childhood. Jounal of Social Issues, 62, 717-36. 


\section{Resumos}

(The crisis of diagnosis in psychiatry and the diagnostic manuals)

It discusses the path taken by psychiatry through diagnostic manuals up until now, with the Research Domain Criteria (RDoC) project, which seeks to achieve therapeutic excellence in psychiatry, by eliminating subjectivity. Four perspectives are presented to think about the diagnosis of psychiatric diseases: diseases that are described in the manuals of psychiatry and that have correspondence in patients in the clinical practice; disorders that are described in the manuals of psychiatry and that generated inaccurate diagnoses; the cultural syndromes and the psychic suffering that are structured subjectively and individually. It is concluded that a therapeutic approach should take into account the complex nature of psychic suffering, being more cautious and considering the individualization with respect to therapeutic approaches.

Keywords: Psychiatry, diagnosis, phenomenology, subjectivity

(La crise du diagnostic en psychiatrie et les manuels de diagnostic)

Cet article analyse le développement de la psychiatrie au moyen de manuels de diagnostic jusqu'à ce jour, prenant comme base le projet Research Domain Criteria $(R D o C)$, qui cherche, en éliminant la subjectivité, à atteindre un niveau d'excellence thérapeutique en psychiatrie. Quatre perspectives sont présentées pour penser le diagnostic des maladies psychiatriques : les maladies décrites dans les manuels de psychiatrie qui correspondent aux patients en pratique clinique; les troubles décrits dans les manuels de psychiatrie qui ont produit des diagnostics inexacts ; les syndromes culturels et la souffrance psychique structurée de façon subjective et individuelle. On conclut qu'une approche thérapeutique devrait tenir compte de la nature complexe de la souffrance psychique, générant un degré plus important de prudence et d'individualisation par rapport aux approches thérapeutiques.

Mots clés: Psychiatrie, diagnostic, phénoménologie, subjectivité

(La crisis del diagnóstico en psiquiatría y los manuales diagnósticos)

A través de los manuales diagnósticos, se discute el camino recorrido por la psiquiatría hasta el momento actual, con el proyecto Research Domain Criteria $(R D o C)$, que busca, eliminando la subjetividad, alcanzar una excelencia terapéutica en la psiquiatría. Se presentan cuatro perspectivas para pensar en el diagnóstico de las enfermedades psiquiátricas: las enfermedades que se describen en los manuales de psiquiatría y que encuentran coincidentes en pacientes en la práctica clínica; los trastornos que se describieron en los manuales de psiquiatría y que generaron diagnósticos imprecisos; los síndromes culturales y el sufrimiento psíquico que está estructurado de forma subjetiva e individual. Se concluye que un abordaje 


\section{OBSERVANDO A PSIQUIATRIA}

terapéutico debe tener en cuenta la naturaleza compleja del sufrimiento psíquico, generando más cautela e individualización en lo que se refiere a los abordajes terapéuticos.

Palabras clave: Psiquiatría, diagnóstico, fenomenología, subjetividad

(Krise der Diagnostik in der Psychiatrie und Diagnosehandbücher)

Dieser Artikel diskutiert den Werdegang der Psychiatrie anhand von Diagnosehandbücher bis zur heutigen Zeit, unter Zuhilfenahme des Projekts Research Domain Criteria (RDoC), dessen Ziel es ist, Subjektivität zu beseitigen um therapeutische Exzellenz in der Psychiatrie zu erreichen. Es werden vier Perspektiven der Diagnose von psychiatrischen Erkrankungen vorgestellt: Krankheiten, die in den Handbüchern der Psychiatrie beschrieben sind und denen von Patienten in der klinischen Praxis entsprechen; Störungen, die in den Handbüchern der Psychiatrie beschrieben wurden und ungenaue Diagnosen erzeugten; kulturelle Syndrome und psychisches Leiden, das subjektiv und individuell strukturiert ist. Schlussfolgernd wird festgehalten, dass der therapeutische Ansatz die komplexe Natur des psychischen Leidens berücksichtigen und eine erhöhten Grad an Aufmerksamkeit und Individualisierung in Bezug auf therapeutische Ansätze aufbringen sollte.

Schlüsselwörter: Psychiatrie Diagnose, Phänomenologie, Subjektivität

Citação/Citation: Pondé, M. P. (2018, março). A crise do diagnóstico em psiquiatria e os manuais diagnósticos. Revista Latinoamericana de Psicopatologia Fundamental, 21(1), 145-166. http://dx.doi.org/10.1590/1415-4714.2018v21n1p145.10

Editores do artigo/Editors: Prof. Dr. Claudio E. Banzato

Recebido/Received: 13.11.2017/ 11.13.2017 Aceito/Accepted: 15.1.2018 / 1.15.2018

Copyright: (C) 2009 Associação Universitária de Pesquisa em Psicopatologia Fundamental/ University Association for Research in Fundamental Psychopathology. Este é um artigo de livre acesso, que permite uso irrestrito, distribuição e reprodução em qualquer meio, desde que o autor e a fonte sejam citados / This is an open-access article, which permits unrestricted use, distribution, and reproduction in any medium, provided the original authors and sources are credited. 


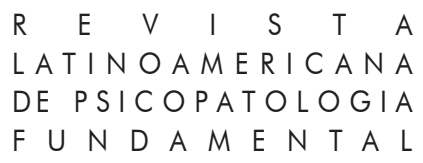

Financiamento/Funding: A autora declara não ter sido financiada ou apoiada / The author has no support or funding funded to report.

Conflito de interesses/Conflict of interest: A autora declara que não há conflito de interesses / The author has no conflict of interest to declare.

Milena Pereira Pondé

Psiquiatra; Pós-Doutorado McGill University (Montreal, Canadá); Professora Adjunta da Escola BAHIANA de Medicina e Saúde Pública (Salvador, BA, Br); Coordenadora do Laboratório Interdisciplinar de Pesquisa em Autismo (LABIRINTO) (Salvador, BA, Br). Av. Dom João VI, 275 - Brotas 40290-000 Salvador, BA, Br.

milenaponde@bahiana.edu.br

This is an open-access article, which permits unrestricted use, distribution, BY-NC and reproduction in any medium for non-commercial purposes provided the original authors and sources are credited. 